LAWRENCE LIVERMORE N A T IO N A L LABORATORY

\title{
X-Ray Line Measurements with High Efficiency Bragg Crystals
}

A. Pak, G. Gregori, J. Knight, K. Campbell, O.L. Landen, S.H. Glenzer

April 26, 2004

15th Topical Conference on High-Temperature Diagnostics San Diego, CA, United States

April 19, 2004 through April 22, 2004 
This document was prepared as an account of work sponsored by an agency of the United States Government. Neither the United States Government nor the University of California nor any of their employees, makes any warranty, express or implied, or assumes any legal liability or responsibility for the accuracy, completeness, or usefulness of any information, apparatus, product, or process disclosed, or represents that its use would not infringe privately owned rights. Reference herein to any specific commercial product, process, or service by trade name, trademark, manufacturer, or otherwise, does not necessarily constitute or imply its endorsement, recommendation, or favoring by the United States Government or the University of California. The views and opinions of authors expressed herein do not necessarily state or reflect those of the United States Government or the University of California, and shall not be used for advertising or product endorsement purposes. 


\title{
$X$ ray line measurements with high efficiency Bragg crystals
}

\author{
A. Pak*, G. Gregori*, J. Knight*, K. Campbell*, O. L. Landen* and S. H. \\ Glenzer* \\ * Lawrence Livermore National Laboratory, PO Box 5508, Livermore, CA 94551
}

\begin{abstract}
We have studied the focusing properties of two highly oriented pyrolitic graphite (HOPG) spectrometers, which differ in the degree of the mosaic spread: ZYA with a low mosaic spread $\left(\gamma=0.4^{\circ}\right)$ and ZYH with a large mosaic spread $\left(\gamma=3.5^{\circ}\right)$. In order to asses the crystal performance for a variety of different experiments, various $\mathrm{K} \alpha$ and $\mathrm{K} \beta \mathrm{x}$-ray lines have been produced using a high-intensity $\left(\gtrsim 10^{17} \mathrm{~W} / \mathrm{cm}^{2}\right)$ short-pulse $(\sim 100 \mathrm{fs})$ laser beam focused onto Ti, V, Zn, and $\mathrm{Cu}$ foils. The measured spectral resolution of the HOPG crystals in both first and second order diffraction has been compared with theoretical predictions. Using known values for the peak reflectivity of HOPG crystals, we have also computed $\mathrm{K} \alpha$ x-ray conversion efficiencies of Ti, V, Zn, and $\mathrm{Cu}$. These results are important to estimate the optimal conditions under which different types of HOPG monochromators can be used for the detection of weak x-ray signals as the one encountered in x-ray Thomson/Compton scattering experiments.
\end{abstract}

\section{INTRODUCTION}

Highly mosaic crystals are of particular interest because their unique crystal plane structure enables them to be highly efficient x-ray diffraction instruments. These type of crystals, for example, have been successfully used in novel x-ray scattering experiments on warm dense matter [1] for x-ray energies $\sim 4.75 \mathrm{keV}$. On the other hand, photon energies $\gtrsim 10 \mathrm{keV}$ are likely to be required for the probing of super-dense states of matter, as the ones encountered in inertial confinement fusion (ICF) experiments [2]. The aim of this work is to characterize the dispersion and resolution of two different types of mosaic crystals and to ascertain their effectiveness for use in x-ray scattering experiments, especially when the detection of very weak signal requires high reflectivity and good spectral resolution.

\section{EXPERIMENT}

The experimental setup is illustrated in Fig. 1. Using the ultra-short pulse (USP) facility at the Lawrence Livermore National Laboratory, a $\sim 400 \mathrm{~mJ}, \lambda=800 \mathrm{~nm}$ laser with pulse length of $100 \mathrm{fs}$ has been used to illuminate $\mathrm{Ti}, \mathrm{V}, \mathrm{Cu}$, and $\mathrm{Zn}$ foils in order to produce $\mathrm{K} \alpha$ and $\mathrm{K} \beta$ x-ray emission lines by electron collision excitation of inner shells. To reduce source broadening the laser was focused to a spot size of $28 \mu \mathrm{m}$. The laser intensity was $\sim 4 \times 10^{17} \mathrm{~W} \mathrm{~cm}^{-2}$. To resolve the $\mathrm{x}$-ray lines we have used high 


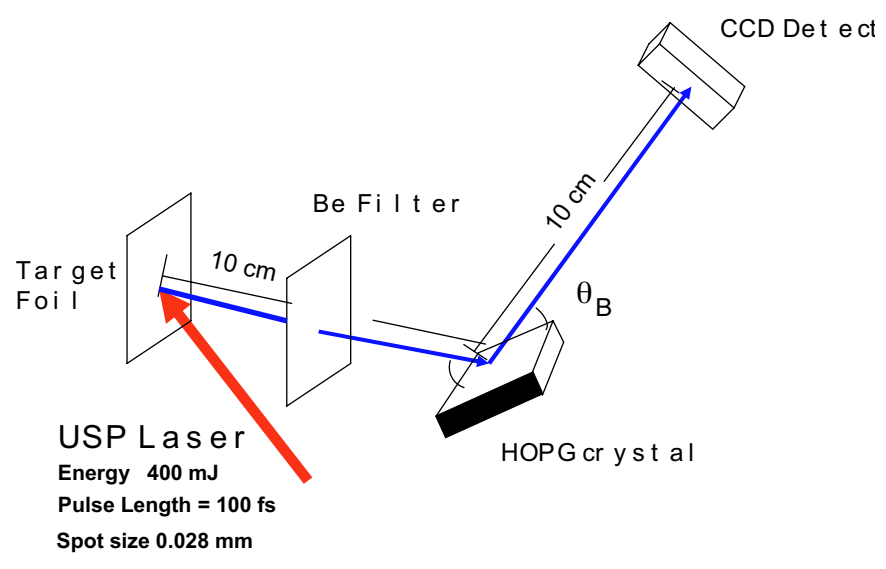

FIGURE 1. Schematic of the experimental setup.

efficiency, highly oriented pyrolitic graphite (HOPG) Bragg crystals. The crystals were used in the mosaic focusing mode, placed at $10 \mathrm{~cm}$ from the foil and the image plane, located at the same distance from the crystal as the x-ray source [3]. A vacuum CCD detector $(256 \times 1024$ array with $26 \mu \mathrm{m}$ pixel size) was used to detect the Bragg scattered photons at the image plane. Low energy photons and visible light were rejected by using a $250 \mu \mathrm{m}$ beryllium filter. Two different types of HOPG crystals have been tested: an Advanced Ceramics ZYA crystal with mosaic spread $\gamma=0.4^{\circ}$ and an Advanced Ceramics ZYH crystal with mosaic spread $\gamma=3.5^{\circ}$. We recall that the mosaic spread $(\gamma)$, is the FWHM of a Gaussian distribution of crystal plane orientations off the normal axis to the surface. As explained, for example, in Ref. [4], the random distribution of scattering planes of the crystal allows a fan of x-rays of the same energy, emitted by a point source, to always find a crystal plane at the correct Bragg angle, and to be refocused on a single point at the image plane. Mosaic focussing is thus achieved. Clearly, a larger mosaic spread crystal would allow, in principle, to find reflecting planes at the correct Bragg angle but separated at larger distances on the crystal surface and thus averaging more over local non-uniformities in crystal Bragg reflectivity. On the other hand, the overall reflectivity of the crystal may be reduced as the mosaic spread is increased if the angular spread subtended by the finite crystal length is not sufficiently large to cover all the possible range of plane orientations. We will discuss both these effects in the following section. Both crystals had the same dimensions of $24 \mathrm{~mm}$ by 24 $\mathrm{mm}$ and a thickness of $2 \mathrm{~mm}$. The separation between the mosaic crystal planes for both crystals is $d=0.3354 \mathrm{~nm}$.

\section{RESULTS AND DISCUSSION}

For mosaic focusing, the Bragg relationship must be satisfied: $n \lambda=2 d \sin \theta_{B}$, where $n$ is the diffraction order, $\lambda$ is the x-ray wavelength, and $\theta_{B}$ is the Bragg angle. By 
differentiating the Bragg's law, we obtain a simple estimate of the crystal dispersion:

$$
\frac{\Delta \lambda}{\lambda}=\frac{\Delta E}{E}=\frac{\Delta \theta}{\tan \theta_{B}}
$$

where $E$ is the $\mathrm{x}$-ray energy and $\Delta \theta$ is the angular spread of the incident x-ray radiation. On the other hand, the spatial distribution of energies (i.e., the dispersion) at a normal image plane is

$$
\frac{\Delta E}{\Delta x}=\frac{E}{2 F \tan \theta_{B}},
$$

with $F=10 \mathrm{~cm}$ is the focal length (source to crystal distance). There are several broadening mechanisms which will affect the focus and resolution of the emission lines. First, finite source size and natural linewidth of the source emitter must be accounted for. For example, for first order diffraction with a Ti $\mathrm{K} \alpha$ line, we have $\Delta E / E \sim 3.1 \times 10^{-4}$ from source broadening and $\Delta E / E \sim 2.1 \times 10^{-4}$ from the natural linewidth of the line [5]. The HOPG has an intrinsic defocusing effect, which is given by the intrinsic rocking curve of Bragg reflections [4], giving $\Delta E / E \sim 1.9 \times 10^{-4}$. Lastly, since a fraction of these high energy photons will penetrate deep $(\gtrsim 100 \mu \mathrm{m})$ into the crystal, there can be a significant volume diffraction which broadens the blue wing side of the diffracted line. Such as volume (depth) broadening is given by

$$
\left(\frac{\Delta E}{E}\right)_{\mathrm{depth}} \approx \frac{\lambda_{m f p}}{2 F} \cos \theta_{B}
$$

where $\lambda_{m f p}$ is the mean free path of the photons in the crystal. For Ti K $\alpha$ we get $\Delta E / E \sim 1.4 \times 10^{-3}$. For the conditions of our experiments we find that this depth effect is the dominant broadening mechanism, and it becomes especially important at higher energies $E \gtrsim 10 \mathrm{keV}$. Crystal surface roughness also has a defocussing effect similar to the depth broadening [6]. It is estimated as $\Delta E / E \sim 1.0 \times 10^{-3}$.

Figure 2 shows the $\mathrm{K} \alpha$ and $\mathrm{K} \beta$ emission spectra for $\mathrm{Ti}, \mathrm{V}, \mathrm{Cu}$ and $\mathrm{Zn}$ foils imaged using a ZYA crystal. In $1^{\text {st }}$ order, with ZYA and ZYH crystals, the measured dispersions for the four $\mathrm{K} \alpha$ energies were within $10 \%$ of the calculated theoretical values. For $\mathrm{Cu}$ and $\mathrm{Zn}$ there are pronounced wings to the higher energy side of their $\mathrm{K} \alpha$ lines due to increasing importance of the volume diffraction mechanism at the higher photon energies. We also notice from the $\mathrm{Zn}$ spectrum line-out a small shoulder on the low energy side of the $\mathrm{K} \alpha$ peak. This point agrees with the position of the lower energy $\mathrm{K} \alpha_{2}$ line, so each $\mathrm{K} \alpha$ line in Figure 2 is actually the convolution of the unresolved $\mathrm{K} \alpha_{1}$ and $\mathrm{K} \alpha_{2}$ lines. As shown in Figure 3, K $\alpha$ images obtained with ZYH crystals appear more uniform than for ZYA crystals, since, as discussed previously, the larger mosaic spread allows X-rays to be diffracted from a larger spatial portion of the crystal, thus averaging over local crystal imperfection. However, the spectral resolution of the ZYH crystal is worse as it is more sensitive to depth broadening than the ZYA, since a given photon will penetrate further on average before meeting a plane at the correct Bragg angle. In Figure 3, we have also compared the $\mathrm{K} \alpha$ and $\mathrm{K} \beta$ spectra of Zn using a ZYA crystal in both $1^{\text {st }}$ and $2^{\text {nd }}$ order diffraction $(n=1$, and $n=2)$. Even in $2^{\text {nd }}$ order we are still unable to 

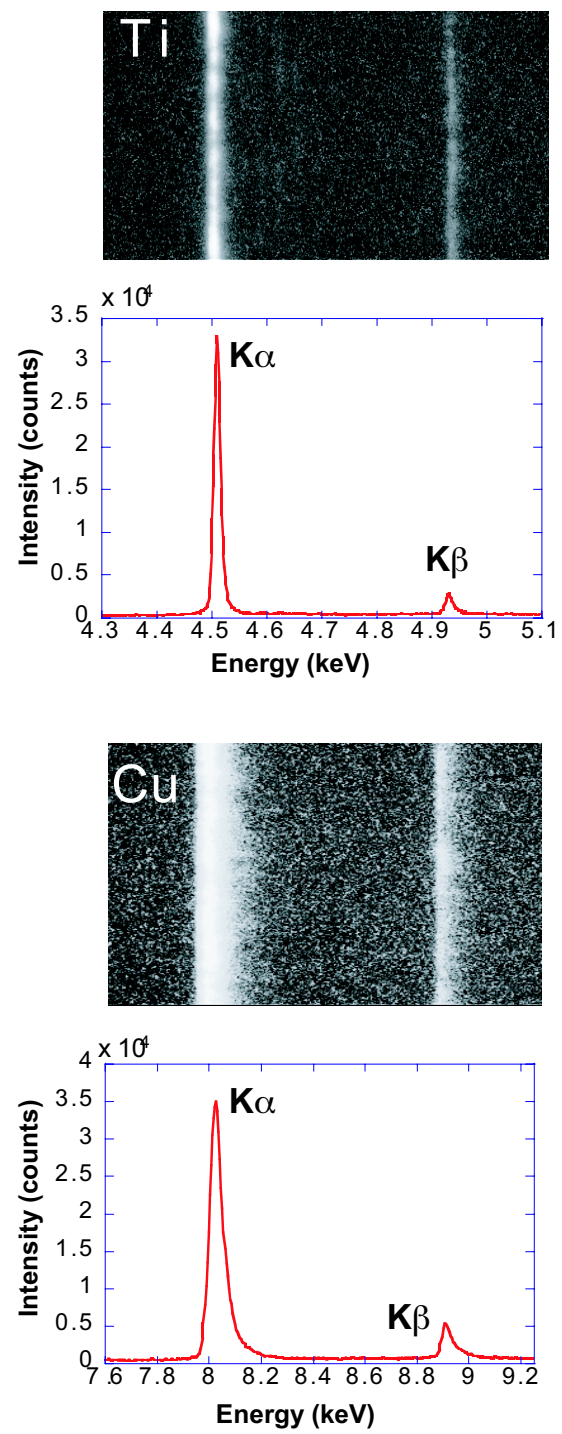
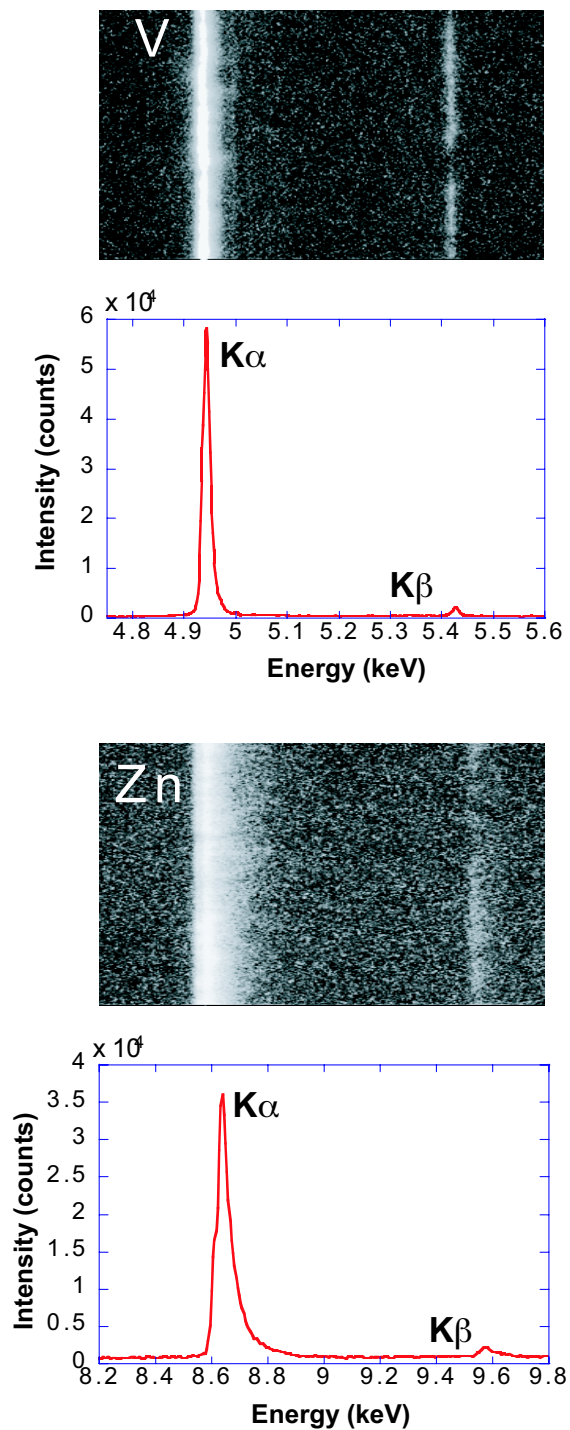

FIGURE 2. $\mathrm{K} \alpha$ and $\mathrm{K} \beta$ emission spectra for $\mathrm{Ti}, \mathrm{V}, \mathrm{Cu}$ and $\mathrm{Zn}$ foils imaged using a $\mathrm{ZYA}$ crystal in $1^{\text {nd }}$ order diffraction. Line-outs are averaged over full spectrum.

clearly resolve the $\mathrm{K} \alpha_{1}$ peak from the $\mathrm{K} \alpha_{2}$ peak, thus confirming the importance of the depth broadening mechanism in determining the measured linewidth (the dependence $\cos \theta_{B}$ given in Eq. 3 confirms that there only is a small change in the line broadening going from first to second order). 

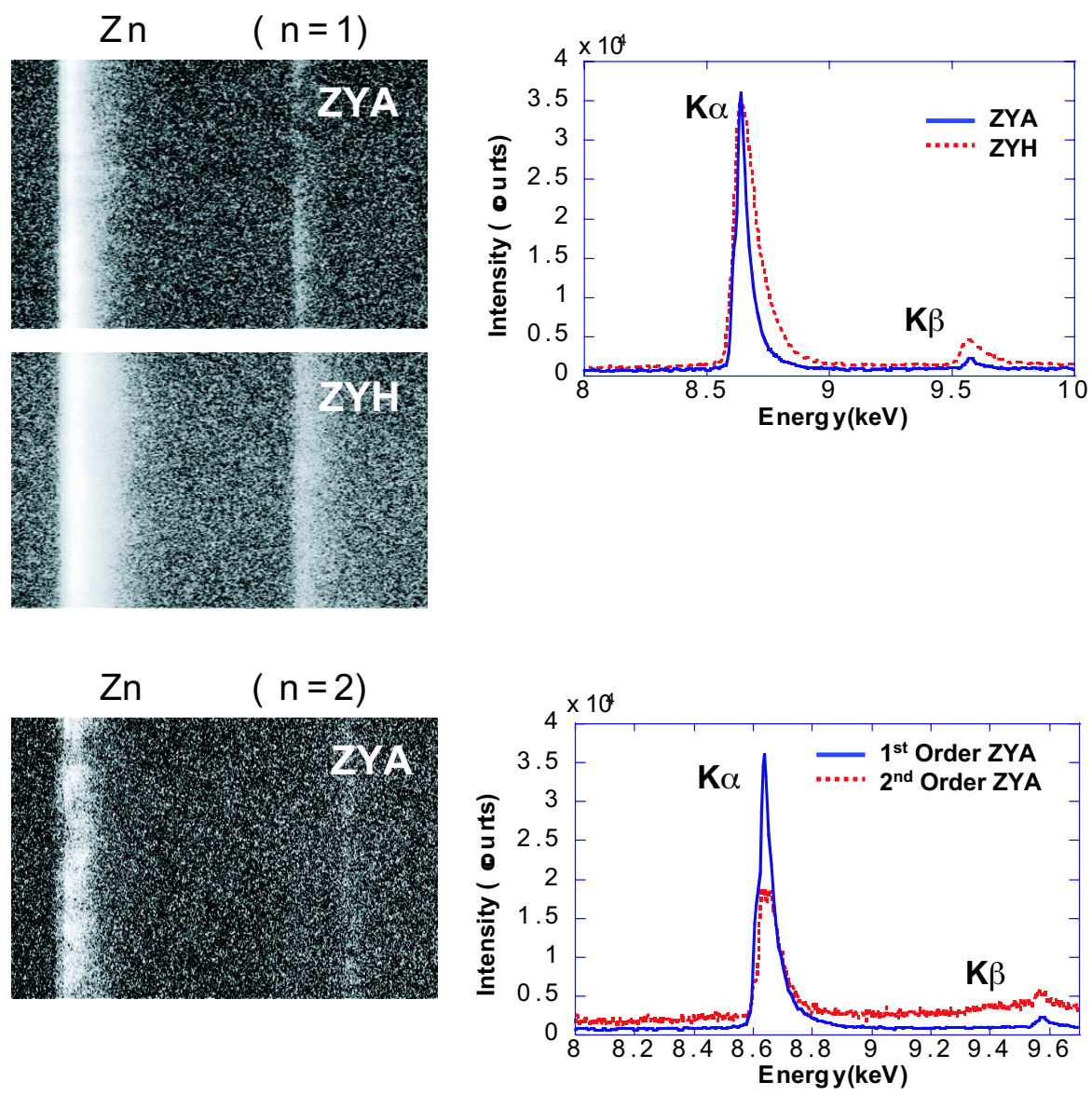

FIGURE 3. $\mathrm{K} \alpha$ and $\mathrm{K} \beta$ emission spectra for $\mathrm{Zn}$ in $1^{\text {st }}$ and $2^{\text {nd }}$ order diffraction, and with $\mathrm{ZYA}$ and ZYH crystals.

Given the total laser energy incident on the foil, we can estimate the corresponding $\mathrm{X}$-ray conversion efficiencies (CE) in the $\mathrm{K} \alpha$ emission line:

$$
\mathscr{C}_{E}=\frac{8 \pi q I_{c t s} g_{C C D} g_{e h} F}{L Q_{E} T_{r} r_{p} \Delta y \theta_{x t a l}}
$$


where $I_{c t s}$ is the total integrated number of CCD counts on the detector integrated over the non-dispersive axis $(\Delta y=6.656 \mathrm{~mm}), q$ is the electric charge, $L$ is the laser energy in the shot, $Q_{E}$ is the quantum efficiency ( $\sim 0.65$ for Ti and V lines, $\sim 0.2$ for $\mathrm{Cu}$ and $\mathrm{Zn}$ lines), $g_{C C D}=3.5$ is the CCD gain (in electrons/counts), $E / g_{e h}=E / 3.6$ is the number of electron-hole pairs produced per incident photon of energy $E, T_{r}$ is the Be filter transmission, and $r_{p}$ is the crystal peak reflectivity. We also have $\theta_{x t a l}=\min (\gamma, \Delta \theta)$, being $\Delta \theta$ the geometrical acceptance angle subtended by the $24 \mathrm{~mm}$ long crystal. Using the tabulated values for ZYA peak reflectivity [7], we were able to obtain estimates of $\mathscr{C}_{E}$ for $\mathrm{Ti}, \mathrm{V}, \mathrm{Cu}$ and $\mathrm{Zn}$. The results are given in Table 1. Also, using these estimates for the conversion efficiency, we can calculate $r_{p}$ values for the ZYA crystal in $2^{\text {nd }}$ order and the ZYH crystal in $1^{\text {st }}$ and $2^{\text {nd }}$ order. These results are also presented in Table 1.

The values of the calculated conversion efficiencies to $\mathrm{K} \alpha$ emission are of the order of $0.01 \%$, which is consistent with previous published work [8]. We find that peak reflectivity of the ZYA crystal are on the same order when used in both $1^{\text {st }}$ or $2^{\text {nd }}$ order, and similarly $r_{p}$ values for the ZYH crystal in $1^{\text {st }}$ and $2^{\text {nd }}$ are also comparable. It is also seen that the $r_{p}$ values for the ZYH crystal in $1^{\text {st }}$ or $2^{\text {nd }}$ order are approximately an order of magnitude less than the peak reflectivity values for the ZYA crystal. The integrated reflectivity $\left(\gamma r_{p}\right)$ of both crystals are $\gtrsim 3 \mathrm{mrad}$, about 30 times higher than for commonly used less mosaic crystals such as LiF or PET [9].

\section{CONCLUSIONS}

Our results show that HOPG crystals can be used to record weak line emission from sub$\mathrm{kJ}$ laser plasmas We have seen that the broadening due to the depth effect is the dominant broadening mechanism at energies above $\sim 9 \mathrm{keV}$, as expected, and it increases with increasing mosaic spread and photon energy. We can therefore conclude that the ZYA crystal with the smaller $\gamma$ provides better resolution of the collected $\mathrm{x}$-rays at higher energies. Also, it was found that the peak reflectivity for the ZYA crystal in $1^{\text {st }}$ order is an order of magnitude greater than the peak reflectivity of the ZYH crystal in the same diffraction order. Measured results also indicate that that the reflectivity of either crystal does not decrease significantly from $1^{\text {st }}$ order when used in $2^{\text {nd }}$ order, and it is of the order of $3 \mathrm{mrad}$. These results seem to confirm that, if high sensitivity is required, ZYA in both first and second order is well suited for the detection of weak signals, as required in x-ray Thomson/Compton scattering experiments to probe dense and warm states of matter [10]. Moreover, the sharpness of the red wing is well suited for diagnostics attempting to resolve the red-shifted plasmon satellite for future collective $\mathrm{x}$-ray scattering experiments.

\section{ACKNOWLEDGMENTS}

This work was performed under the auspices of the U.S. Department of Energy by the University of California Lawrence Livermore National Laboratory under Contract No. 
W-7405-ENG-48. We also acknowledge support from Laboratory Directed Research and Development grant No. 02-ERD-13.

\section{REFERENCES}

1. Glenzer, S. H., Gregori, G., Lee, R. W., Rogers, F. J., Pollaine, S. W., and Landen, O. L., Phys. Rev. Lett., 90, 175002 (2003).

2. Lindl, J. D., Inertial Confinement Fusion, Springer-Verlag, New York, 1998.

3. Glenzer, S. H., Gregori, G., Rogers, F. J., Froula, D. H., Pollaine, S. W., Wallace, R. S., and Landen, O. L., Phys. Plasmas, 10, 2433 (2003).

4. Zachariasen, W. H., X-ray Diffraction in Crystals, Wiley, New York, 1945.

5. Krause, M. O., and Oliver, J. H., J. Phys. Chem. Ref. Data, 8, 329 (1979).

6. Ice, G. E., and Sparks, C. J., Nucl. Instrument. Methods Phys. Res., A291, 110 (1990).

7. Freund, A. K., Munkholm, A., and Brennan, S., SPIE, 2865, 68 (1996).

8. Reich, C., Gibbon, P., Uschmann, I., and Förster, E., Phys. Rev. Lett., 84, 4846 (2000).

9. Marshall, F. J., and Oertel, J. A., Rev. Sci. Instrum., 68, 735 (1997).

10. Gregori, G., Glenzer, S. H., Rozmus, W., Lee, R. W., and Landen, O. L., Phys. Rev. E, 67, 026412 (2003). 


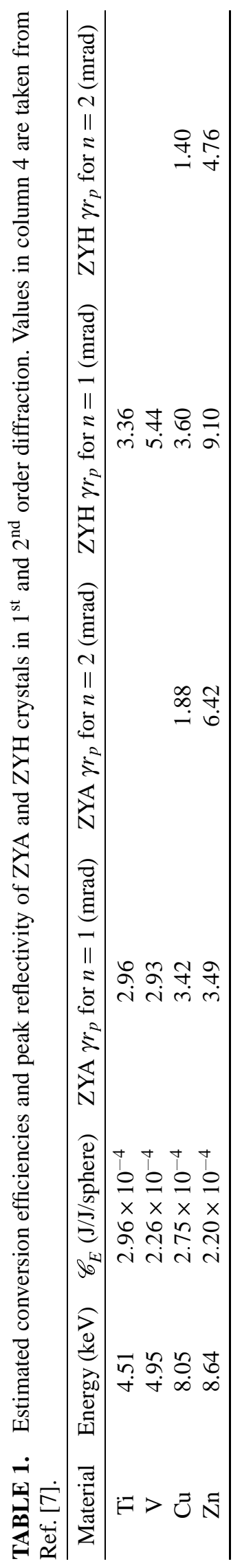

